MINI-REVIEW

\title{
Dehydroepiandrosterone replacement therapy
}

\author{
Eleanor M Gurnell and V Krishna K Chatterjee \\ Department of Medicine, University of Cambridge, Addenbrooke's Hospital, Hills Road, Cambridge CB2 2QQ, UK \\ (Correspondence should be addressed to V K K Chatterjee, Department of Medicine, University of Cambridge, Level 5, Addenbrooke's Hospital, Hills Road, \\ Cambridge CB2 2QQ, UK; Email: kkc1@mole.bio.cam.ac.uk)
}

\section{Introduction}

Dehydroepiandrosterone (DHEA) and its sulphated derivative DHEAS are the most abundant circulating steroids, and are derived from the zona reticularis of the adrenal cortex. The foetal adrenals synthesise significant quantities of DHEA, but production of this steroid falls postnatally. The onset of adrenarche (between the ages of 6 and 8 years) is characterised by increasing biosynthesis of DHEA, and circulating DHEA(S) levels reach a peak in the third decade of life (1). Thereafter, there is an inexorable decline in DHEA production, such that, by the age of 80 years, subjects show DHEA levels that are only $10-20 \%$ of those in young adults (2); circulating cortisol levels, however, remain relatively unchanged.

The physiological role of DHEA is not completely understood. DHEA is a substrate for androstenedione and testosterone synthesis, and adrenarche is accompanied by pubic and axillary hair development, suggesting that one of the actions of DHEA is as an adrenal androgen. However, there is evidence to suggest that it may also exert effects in the central nervous system. It is an antagonist at the gamma amino butyric acid receptor and an agonist at the NMDA receptor (3). It also opposes the action of glucocorticoids, antagonising glucocorticoid-induced thymic involution or corticosterone neurotoxicity in the hippocampus $(4,5)$. However, to date, a specific receptor which mediates the action of DHEA has not been identified - and it remains to be determined as to whether it acts at the cell surface or intracellularly.

Unfortunately, studies of DHEA administration in non-primate species are of limited value for two reasons: first, there is no rise in DHEAS levels with adrenarche; secondly, there is no age-related decline in circulating levels of this steroid. Here, we review clinical studies of DHEA treatment in humans in two contexts: (i) as a supplement for normal elderly subjects, to reverse the age-related decline in circulating DHEA levels; and (ii) replacement in patients with adrenal insufficiency and who have a near-absolute deficiency of this steroid.

\section{DHEA replacement in ageing}

As a consequence of either diminished adrenal $17 \alpha-$ hydroxylase enzyme activity or involution of the zona reticularis, a number of studies have documented an age-related decline in DHEAS levels. In both sexes, there is a reduction of about $2 \%$ per year (6), but the absolute levels are lower in women than in men in the 50 to 89year age range (7). Furthermore, cross-sectional studies have documented an association between the decline in DHEAS levels and various adverse effects of ageing. Barrett-Connor et al. (8) showed an inverse relationship between DHEAS levels and cardiovascular disease and mortality in elderly men, but this relationship was not observed in women (9). Low DHEAS levels have been correlated with heightened risk of breast cancer in premenopausal women (10). There is a positive correlation between reduced serum DHEAS levels and lower bone mineral density in the spine, hip and radius in women aged 45-69 years (11), but no such effect is noted in men (12). In the central nervous system, lower DHEAS levels are associated with depressed mood in older women but not men (13), and a higher cortisol/ DHEA ratio correlates with cognitive decline in both sexes (14). There are conflicting reports of lower DHEAS levels in Alzheimer's disease $(15,16)$. DHEAS levels are lower in men with type 2 diabetes mellitus (17), and insulin-sensitising drug therapy enhances DHEAS levels, suggesting an inverse correlation with insulin resistance. However, a major concern in relation to such epidemiological data is that falling DHEAS levels may simply be a marker of the ageing process, tracking the morbidities associated with it, rather than being causally related. Accordingly, a number of workers have sought to show changes in biochemical, metabolic and neuropsychological parameters following DHEA supplementation in elderly subjects.

Pharmacokinetic studies have established an appropriate dose range for DHEA replacement in ageing. Arlt et al. (18) showed that $50 \mathrm{mg}$ oral DHEA in men aged 49-70 years restored circulating DHEAS to youngadult levels, whereas a $100 \mathrm{mg}$ daily dose was supraphysiological. More recently, administration of 25 or $50 \mathrm{mg}$ DHEA in elderly men and women achieved steady-state physiological levels in 8 days (19). Interestingly, the half-life of circulating DHEA was more than $20 \mathrm{~h}$, rather like its longer-lived sulphated metabolite, suggesting that there may be significant back-conversion of DHEAS in vivo. Circulating testosterone and oestrogens also rose following DHEA supplementation, albeit to levels within the youngadult range. 
A randomised, double-blind, placebo-controlled trial examined the effect of $50 \mathrm{mg}$ DHEA treatment for 6 months in 13 men and 17 women aged 40-70 years (20). In addition to the restoration of young-adult DHEA(S) levels, there was a marked increase in physical and psychological well-being in both sexes ( $67 \%$ of men and $81 \%$ of women), but no change in libido. This effect was associated only with increased insulin-like growth factor-I and reduced insulin-like growth factor-binding protein-1 levels, but there were no changes in circulating sex hormone binding globulin (SHBG), oestrogens or lipids. Extending these observations, the same group have studied $100 \mathrm{mg}$ of DHEA replacement for 6 months in elderly subjects (9 men and 10 women; 50-65 years) (21); they showed restoration of circulating DHEAS and the cortisol/ DHEAS ratio to young-adult levels. There was a decrease in fat mass and an enhancement of muscle strength in men but not in women. Circulating androgens (androstenedione, testosterone, dihydrotestosterone) rose above young-adult levels in women but not in men, suggesting that there are gender-specific differences in the response to DHEA administration. However, another study involving the administration of $100 \mathrm{mg}$ DHEA for 3 months in elderly men showed no effect on body composition, serum prostate-specific antigen or urological function (22).

Most recently, Baulieu et al. have published the results of a randomised, placebo-controlled study of $50 \mathrm{mg}$ DHEA replacement for 12 months in 280 men and women aged 60-79 years (23). Whilst youngadult DHEAS levels were restored in both sexes, gender differences were again observed for other parameters. Serum testosterone and androstandiol glucuronide rose to slightly supraphysiologic levels at 6 months in $21 \%$ of women but not in men. Bone mineral density improved at the femoral neck (in the 60-69-year group) and radius (in the 70-79-year group), and there was a fall (selectively in women) in the levels of serum collagen telopeptide. Likewise, only women reported an increase in libido and sexual function and satisfaction. The following skin changes were seen in both sexes: increased skin hydration, diminished facial pigmentation and epidermal atrophy; enhanced sebum production (particularly in women more than 70 years of age). There were no changes in vascular function, as assessed by ultrasonographic methods.

Following the menopause, testosterone and androstenedione levels fall by $50 \%$, and the decline in DHEAS with ageing leads to a further fall in circulating androgens. This knowledge has prompted treatment of postmenopausal women with DHEA as a form of androgen replacement. Labrie et al. have successfully treated 14 women aged 60-70 years for 12 months with 10\% DHEA cream applied topically; serum DHEA levels rose 10-fold (24). In conjunction with this, these workers observed increased sebum production and an oestrogenic effect on vaginal epithelium (increasing its maturation index). Bone mineral density at the hip was enhanced, there being increased osteoblastic (osteocalcin) and reduced osteoclastic (bone alkaline phosphatase, urinary hydroxyproline) marker activity. Other changes included a $10 \%$ reduction in skin-fold thickness, lower blood glucose and insulin levels, without any adverse effects on the lipid profile (25). Subjects also reported improved well-being. In contrast, $50 \mathrm{mg}$ DHEA administered orally in 60 perimenopausal women (45-55 years) had a lesser effect (26): despite a twofold rise in DHEAS levels, there was no effect on mood, cognition, quality of life, or libido.

\section{DHEA replacement in adrenal insufficiency}

Circulating DHEAS levels in adrenal failure are very low or undetectable relative to those present in the context of ageing. Such deficiency is observed in secondary as well as primary adrenal insufficiency (Addison's disease), implying that production of this adrenal steroid (like the glucocorticoid production) is pituitary-dependent. Whilst glucocorticoid and mineralocorticoid deficiencies in adrenal insufficiency are lifethreatening and require oral replacement, the associated failure of DHEA synthesis is not usually corrected. On the other hand, despite optimal therapy with conventional steroids, patients with Addison's disease report persistent fatigue and reduced well-being $(27,28)$. These observations have prompted recent trials of DHEA replacement.

In 10 patients with hypopituitarism, Young et al. (29) showed that $50 \mathrm{mg}$ DHEA replacement restored DHEAS, androstenedione and testosterone to youngadult levels, whereas a $200 \mathrm{mg}$ daily dose was supraphysiologic. Arlt et al. (30) examined DHEA replacement in young volunteers whose endogenous adrenal steroidogenesis had been suppressed with dexamethasone; again, a $50 \mathrm{mg}$ daily dose was found to be appropriate, whereas $100 \mathrm{mg}$ induced supraphysiologic concentrations of DHEAS.

A randomised, double-blind, placebo-controlled trial of $50 \mathrm{mg}$ DHEA replacement was conducted with 24 women with adrenal (14 primary, 10 secondary) insufficiency (31). Physiological levels of DHEA(S) and androstenedione were restored, and serum testosterone levels rose from below to within the low normal range. Following 4 months of therapy, the subjects showed a significant reduction in scores for depression and anxiety (as assessed by psychological testing), together with improvements in overall well-being and mood. The patients also reported markedly increased sexual thoughts/interest, together with enhanced mental and physical sexual satisfaction. Serum testosterone rose from below to within the low normal range, showing a fall in SHBG, and 19 subjects developed some cutaneous androgenic side-effects. A later study of DHEA replacement in nine women with Addison's 
disease also reported increased apocrine sweat secretion and acne, there being no differences between the daily dosages of $50 \mathrm{mg}$ and $200 \mathrm{mg}$ (32).

We undertook a randomised, placebo-controlled trial in both men $(n=15)$ and women $(n=24)$ with Addison's disease (33). Following 3 months of treatment with $50 \mathrm{mg}$ DHEA, a similar restoration of DHEAS and androstenedione levels was observed in both sexes; there was a rise in serum testosterone and a fall in SHBG in women but not in men. There was a tendency for improved overall well-being scores in the General Health Questionnaire, and significant improvement in self-esteem. Mood and fatigue also improved significantly, the benefits being evident in the evenings. We found no effects on cognitive or sexual function. No changes in body composition or bone density were seen, albeit after relatively short-term hormone replacement. In view of the beneficial psychological effects in males, independent of changes in circulating testosterone, we concluded that DHEA might act directly on the central nervous system rather than via enhanced peripheral androgen biosynthesis. No significant adverse effects were seen in our patients.

\section{Conclusions}

On the basis of current evidence, it is clear that DHEA supplementation in ageing subjects is biochemically effective at restoring young-adult levels of DHEAS and normalising the circulating cortisol/DHEA ratio. Even though it may raise circulating androgens to slightly supraphysiologic levels in women, this does not appear to be associated with significant adverse cutaneous (hirsutism, acne) or metabolic (lipid-profile) effects. Interestingly, some of its beneficial effects, for example on bone mineral density and sexual function, are gender-specific or more evident in women, which suggests that the agent may be acting as a precursor for androgen or oestrogen biosynthesis. In future studies of postmenopausal women, it would be of interest to compare the efficacy of testosterone replacement directly with DHEA treatment. In both sexes, significant psychological benefits and enhanced wellbeing have been documented. Whilst these observations may justify short-term treatment, longer-term studies are now required to determine whether, as epidemiological studies suggest, cardiovascular morbidity and mortality, cognitive decline or cancer risk can be influenced by DHEA replacement.

Short-term replacement studies suggest that there are clear-cut beneficial effects of DHEA replacement on well-being, mood and sexual function in adrenal insufficiency. Longer-term controlled studies are now needed to examine whether such improvements are sustained. As the psychological benefits have been documented for both sexes, it is important to continue evaluating the effect of DHEA in men as well as in women. Future, larger studies may be able to determine whether certain effects (e.g. on sexual function) are maximal in subgroups (e.g. postmenopausal women) or if they are influenced by concurrent, conventional hormone-replacement therapy. An advantage of studying DHEA replacement in primary rather than secondary insufficiency is that it avoids any confounding effects of other pituitary (e.g. growth hormone) deficiencies. It is also recognised that patients with Addison's disease have reduced bone mineral density (BMD) which correlates with the duration and dosage of glucocorticoid replacement $(34,35)$. If DHEA has an antiglucocorticoid effect $(4,5)$, longer-term studies will determine whether replacement therapy can augment $\mathrm{BMD}$, as in ageing, and whether there are beneficial changes in body composition.

Exposure to DHEA augments natural killer cellmediated cytotoxicity in vitro (36) and DHEA supplementation increases natural killer cell numbers (37). Although DHEA treatment does not influence the immune response to influenza vaccination (38), future studies of immune function following DHEA replacement might be revealing, particularly those which examine better in vivo immunological correlates of its activity.

Finally, studies of DHEA replacement might point to a pharmacological role for DHEA in certain contexts. Although short-term (2-week) placebo-controlled studies have shown no effect of DHEA treatment on cognitive function in the elderly (39), a recent study has shown that DHEA therapy for 6 weeks has a significant beneficial effect on major depression (40).

\section{References}

1 Ibanez L, Dimartino-Nardi J, Potau N \& Saenger P. Premature adrenarche - normal variant or forerunner of adult disease? Endocrine Reviews 200021 671-696.

2 Vermeulen A. Dehydroepiandrosterone sulfate and aging. Annals of the New York Academy of Sciences 1995774 121-127.

3 Majewska MD. Neuronal actions of dehydroepiandrosterone. Possible roles in brain development, aging, memory, and affect. Annals of the New York Academy of Sciences 1995774 111-120.

4 May M, Holmes E, Rogers W \& Poth M. Protection from glucocorticoid induced thymic involution by dehydroepiandrosterone. Life Science 199146 1627-1631.

5 Kimonides VG, Spillantini MG, Sofroniew MV, Fawcett JW \& Herbert J. Dehydroepiandrosterone (DHEA) antagonises the neurotoxic effects of corticosterone and translocation of SAPK3 in hippocampal primary cultures. Neuroscience $199989429-436$.

6 Orentreich N, Brind JL, Rizer RL \& Vogelman JH. Age changes and sex differences in serum dehydroepiandrosterone sulfate concentrations throughout adulthood. Journal of Clinical Endocrinology and Metabolism 198459 551-555.

7 Laughlin GA \& Barrett-Connor E. Sexual dimorphism in the influence of advanced aging on adrenal hormone levels: the Rancho Bernardo Study. Journal of Clinical Endocrinology and Metabolism 200085 3561-3568.

8 Barrett-Connor E, Khaw K-T \& Yen SSC. A prospective study of dehydroepiandrosterone sulfate, mortality and cardiovascular disease. New England Journal of Medicine 1986315 1519-1524.

9 Barrett-Connor E \& Goodman-Gruen D. Dehydroepiandrosterone sulfate does not predict cardiovascular death in postmenopausal women. The Rancho Bernardo Study. Circulation 199591 $1757-1760$. 
10 Helzlsouer KJ, Gordon GB, Alberg AJ, Bush TL \& Comstock GW. Relationship of prediagnostic serum levels of dehydroepiandrosterone and dehydroepiandrosterone sulfate to the risk of developing premenopausal breast cancer. Cancer Research 1992 52 1-4.

11 Szathmari M, Szucs J, Feher T \& Hollo I. Dehydroepiandrosterone sulphate and bone mineral density. Osteoporosis International $1994484-88$.

12 Greendale GA, Edelstein S \& Barrett-Connor E. Endogenous sex steroids and bone mineral density in older women and men: the Rancho Bernardo Study. Journal of Bone Mineral Research 1997 12 1833-1843.

13 Barrett-Connor E, von Muhlen D, Laughlin GA \& Kripke A. Endogenous levels of dehydroepiandrosterone sulfate, but not other sex hormones, are associated with depressed mood in older women: the Rancho Bernardo Study. Journal of the American Geriatrics Society $1999 \mathbf{4 7}$ 685-691.

14 Kalmijn S, Launer LJ, Stolk RP, de Jong FH, Pols HAP, Hofman A et al. A prospective study on cortisol, dehydroepiandrosterone sulfate and cognitive function in the elderly. Journal of Clinical Endocrinology and Metabolism $1998 \mathbf{8 3} 3487-3492$.

15 Nasman B, Olsson T, Backstrom T, Eriksson S, Grankvist K, Viitanen $\mathrm{M}$ et al. Serum dehydroepiandrosterone sulfate in Alzheimer's disease and in multi-infarct dementia. Biological Psychiatry 199130 684-690.

16 Schneider LS, Hinsey M \& Lyness S. Plasma dehydroepiandrosterone sulfate in Alzheimer's disease. Biological Psychiatry 1992 31 205-208.

17 Barrett-Connor E. Lower endogenous androgen levels and dyslipidemia in men with non-insulin-dependent diabetes mellitus. Annals of Internal Medicine 1992117 807-811.

18 Arlt W, Haas J, Callies F, Reincke M, Hubler D, Oettel M et al. Biotransformation of oral dehydroepiandrosterone in elderly men: significant increase in circulating estrogens. Journal of Clinical Endocrinology and Metabolism 199984 2170-2176.

19 Legrain S, Massien C, Lahlou N, Roger M, Debuire B, Diquet B et al. Dehydroepiandrosterone replacement administration: pharmacokinetic and pharmacodynamic studies in healthy elderly subjects. Journal of Clinical Endocrinology and Metabolism 200085 3208-3217.

20 Morales AJ, Nolan JJ, Nelson JC \& Yen SSC. Effects of replacement dose of dehydroepiandrosterone in men and women of advancing age. Journal of Clinical Endocrinology and Metabolism $1994 \mathbf{7 8}$ $1360-1367$.

21 Morales AJ, Haubrich RH, Hwang JY, Asakura H \& Yen SSC. The effect of six months treatment with a $100 \mathrm{mg}$ daily dose of dehydroepiandrosterone (DHEA) on circulating sex steroids, body composition and muscle strength in age-advanced men and women. Clinical Endocrinology 199849 421-432.

22 Flynn MA, Weaver-Osterholtz D, Sharpe-Timms KL, Allen S \& Krause G. Dehydroepiandrosterone replacement in aging humans. Journal of Clinical Endocrinology and Metabolism 1999 84 1527-1533.

23 Baulieu EE, Thomas G, Legrain S, Lahlou N, Roger M, Debuire B et al. Dehydroepiandrosterone (DHEA), DHEA sulfate, and aging: contribution of the DHEAge Study to a sociobiomedical issue. PNAS 200097 4279-4284.

24 Labrie F, Diamond P, Cusan L, Gomez JL, Belanger A \& Candas B. Effect of 12-month dehydroepiandrosterone replacement therapy on bone, vagina and endometrium in postmenopausal women. Journal of Clinical Endocrinology and Metabolism 199782 34983505.

25 Diamond P, Cusan L, Gomez J-L, Belanger A \& Fabrie F. Metabolic effects of 12-month percutaneous dehydroepiandrosterone replacement therapy in postmenopausal women. Journal of Endocrinology 1996150 S43-S50.

26 Barnhart KT, Freeman E, Grisso JA, Rader DJ, Sammel M, Kapoor $\mathrm{S}$ et al. The effect of dehydroepiandrosterone supplementation to symptomatic perimenopausal women on serum endocrine profiles, lipid parameters, and health-related quality of life. Journal of Clinical Endocrinology and Metabolism 199984 3896-3902.

27 Reidel M, Weise A, Schurmeyer TH \& Brabant G. Quality of life in patients with Addison's disease: effects of different cortisol replacement modes. Experimental Clinical Endocrinology 1993 101 106-111.

28 Baker SJK, Hunt PJ \& Wass JAH. Assessing the potential for finetuning the management of Addison's disease/steroid replacement therapy. Journal of Endocrinology 1997155 (Suppl) P2 (Abstract).

29 Young J, Couzinet B, Nahoul K, Brailly S, Chanson P, Baulieu EE et al. Panhypopituitarism as a model to study the metabolism of dehydroepiandrosterone (DHEA) in humans. Journal of Clinical Endocrinology and Metabolism 199782 2578-2585.

30 Arlt W, Justl H-G, Callies F, Reincke M, Hubler D, Oettel M et al. Oral dehydroepiandrosterone for adrenal androgen replacement: pharmacokinetics and peripheral conversion to androgens and estrogens in young females after dexamethasone suppression. Journal of Clinical Endocrinology and Metabolism $1998 \mathbf{8 3} 1928$ 1934.

31 Arlt W, Callies F, van Vlijmen JC, Koehler I, Reincke M, Bidlingmaier $\mathrm{M}$ et al. Dehydroepiandrosterone replacement in women with adrenal insufficiency. New England Journal of Medicine 1999341 1013-1020.

32 Gebre-Medhin G, Husebye ES, Mallmin H, Helstrom L, Berne C, Karlsson FA et al. Oral dehydroepiandrosterone (DHEA) replacement therapy in women with Addison's disease. Clinical Endocrinology $2000 \mathbf{5 2}$ 775-780.

33 Hunt PJ, Gurnell EM, Huppert FA, Richards C, Prevost AT, Wass JA et al. Improvement in mood and fatigue after dehydroepiandrosterone replacement in Addison's disease in a randomized, double blind trial. Journal of Clinical Endocrinology and Metabolism 200085 4650-4656.

34 Zelissen PMJ, Croughs RJM, van Rijk PP \& Raymakers JA. Effect of glucocorticoid replacement therapy on bone mineral density in patients with Addison disease. Annals of Internal Medicine 1994 120 207-210.

35 Heureux F, Maiter D, Boutsen Y, Devogelaer JP, Jamart J \& Donckier J. Evaluation of glucocorticoid replacement therapy and of their effects on bone mineral density in Addison's disease. Annals of Endocrinology 200061 179-183.

36 Solerte SB, Fioravanti M, Vignati G, Giustina A, Cravello L \& Ferrari E. Dehydroepiandrosterone sulfate enhances natural killer cell cytotoxicity in humans via locally generated immunoreactive insulin-like growth factor I. Journal of Clinical Endocrinology and Metabolism 199984 3260-3267.

37 Casson PR, Andersen RN, Herrod HG, Stentz FB, Straughn AB, Abraham GE et al. Oral dehydroepiandrosterone in physiologic doses modulates immune function in postmenopausal women. American Journal of Obstetrics and Gynecology $19931691536-$ 1539.

38 Danenberg HD, Ben-Yehuda A, Zakay-Rones Z, Gross DJ \& Friedman G. Dehydroepiandrosterone treatment is not beneficial to the immune response to influenza in elderly subjects. Journal of Clinical Endocrinology and Metabolism 199782 2911-2914.

39 Wolf OT \& Kirschbaum C. Wishing a dream came true: DHEA as a rejuvenating treatment? Journal of Endocrinological Investigation 199821 133-135.

40 Wolkowitz OM, Reus VI, Keebler A, Nelson N, Friedland M, Brizendine L et al. Double-blind treatment of major depression with dehydroepiandrosterone. American Journal of Psychiatry $1999156646-649$.

Received 23 February 2001

Accepted 27 April 2001 